\title{
Formal Constraints on Metarules*
}

Stuart M. Shieber, Susan U. Stucky, Hans Uszkoreit, and Jane J. Robinson

\author{
SRI International \\ 333 Ravenswood Avenue \\ Menlo Park, California
}

\begin{abstract}
Metagrammatical formalisms that combine context-free phrase structure rules and metarules (MPS grammars) allow concise statement of generalizations about the syntax of natural languages. Unconstrained MPS grammars, unfortunately, are not computationally "safe." We evaluate several proposals for constraining them, basing our assessment on computational tractability and explanatory adequacy. We show that none of them satisfies both criteria, and suggest new directions for research on alternative metagrammatical formalisms.
\end{abstract}

\section{Introduction}

The computational-linguistics community has recently shown interest in a variety of metagrammatical formalisms for encoding grammars of natural language. A common technique found in these formalisms involves the notion of a metarule, which, in its most common conception, is a device used to generate grammar rules from other given grammar rules. ${ }^{1}$ A metarule is essentially a statement declaring that, if a grammar contains rules that match one specified pattern, it also contains rules that match some other specified pattern. For example, the following metarule

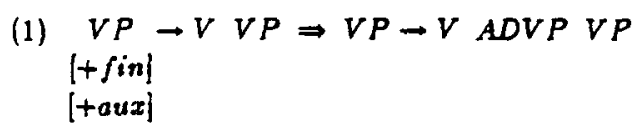

states that, if there is a rule that expands a finite $V P$ into a finite auxiliary and a nonfinite $V P$, there will also be a rule that expands the $V P$ as before except for an additional adverb between the auxiliary and the nonfinite VP. ${ }^{2}$ The patterns may contain variables, in which case they characterize "families" of related rules rather than individual pairs.

"This research was supported by the National Seience Foundation grant No. IST-8103550. The view: and conclusions expressed in this document are those of the authors and should not be interpreted as representative of the views of the National Science Foundation or the United States gorernment. We are indebted to Fernando Pereira, Stanley Peters, and Stanley Rosensehein for many helpful discussions leading to the writing of this paper.

${ }^{1}$ Metarules were first utilized for natural-language research and are most extensirely developed within the theory of Generalized Phrace Structure Grammar (GPSG) [Gazdar and Pullum, 1982; Gawron et ah, 1982; Thompson, 1882|.

${ }^{2}$ metarule similar to our example was proposed by Gazdar, Pullum, and Sag [1982, p. 607].
The metarule notion is a seductive one, intuitively allowing generalizations about the grammar of a language to be stated concisely. However, unconstrained metarule formalisms may possess more expressive power than is apparently needed, and, moreover, they are not always computationally "safe." For example, they may generate infinite sets of rules and describe arbitrary languages. In this paper we examine both the formal and linguistic implications of various constraints on metagrammatical formalisms consisting of a combination of context-free phrase structure rules and metarules, which we will call metarule phrase-structure (MPS) grammars.

The term "MPS grammar" is used in two ways in this paper. An MPS grammar can be viewed as a grammar in its own right that characterizes a language directly. Alternatively, it can be viewed as a melogrammar, that is, as a generator of a phrase structure object grammar, the characterized language being defined as the language of the object grammar.

Uszkoreit and Peters [1982] have developed a formal definition of MPS grammars and have shown that an unconstrained MPS grammar can encode any recursively enumerable language. As long as the framework for grammatical description is not seen as part of a theory of natural language, this fact may not affect the usefulness of MPS grammars as tools for purely descriptive linguisties research; however, it has direct and obvious impact on those doing research in a computational or theoretical linguistic paradigm. Clearly, some way of constraining the power of MPS grammars is necessary to enable their use for encoding grammars in a computationally feavible way. In the sections that follow, we consider several formal proposals for constraining their power and discuss some of their computational and linguistic ramifications.

In our discussion of the computational ramifications of the proposed constraints, we will use the notion of weak-generative capacity as a barometer of the expressive power of a formalism. Other notions of expressivity are possible, although some of the traditional ones may not be applicable to MPS grammars. Strong-generative capacity, for instance, though well-defined, seems to be an inadequate notion for comparison of MPS grammars, since it would have to be extended to include informa tion about rule derivations as well as tree derivations. Similarly, we do not mean to imply by our arguments that the class of natural languages corresponds to some class that ranks low in the Chomsky hierarchy merely because the higher classes are less constrained in weak-generative power. The appropriate characterization of possible natural languages may not coincide at all 
with the divisions in the Chomsky hierarchy. Nevertheless weakgenerative capacity - the weakest useful metric of capacity-will be the primary concern of this paper as a well-defined and relevant standard for measuring constraints.

\section{Constraints by Change of Perspective}

Peters and Ritchie [1973) have pointed out that contextsensitive grammars have no more than context-free power when their rules are viewed as node-admissibility conditions. This suggests that MPS grammars might be analogously constrained by regarding the metarules as something other than phrasestructure grammar generators. A brief examination of three alternative approaches indicates, however, that none of them clearly yieids any useful constraints on weak-generative capacity. Two of the alternatives discussed below consider metarules to be part of the grammar itself, rather than as part of the metagrammar. The third views them as a set of redundant generalizations about the grammar.

Stucky [fortheoming] investigates the possibility of defining metarules as complex node-admissibility conditions, which she calls meta-node-admissibility conditions. Two computationally desirable results could ensue, were this reinterpretation possible. Because the metarules do not generate rules under the metanode-admissibility interpretation, it follows that there will be neither a combinatorial explosion of rules nor any derivation resulting in an infinite set of rules (both of which are potential problems that could arise under the original generative interpretation).

For this reinterpretation to have a computationally tractable implementation, however, two preconditions must be met. First, an independent mechanism must be provided that assigas to any string a finite set of trees, including those admitted by the metarules together with the base rules. Second, a procedure must be defined that checks node admissibilities according to the base rules and metarules of the grammar-and that terminates. It is this latter condition that we suspect will not be possible without constraining the weak-generative capacity of MPS grammars. Thus, this perspective does not seem to change the basic expressivity problems of the formalism by itself.

A second alternative, proposed by Kay [1982], is one in which metarules are viewed as chart-manipulating operators on a chart parser. Here too, the metarules are not part of a metagrammar that generates a context-free grammar; rather, they constitute a second kind of rule in the grammar. Just like the meta-node-admissibility interpretation, Kay's explication seems to retain the basic problem of expressive power, though Kay hints at a gain in efficiency if the metarules are compiled into a finite-state transducer.

Finally, an alternative that does not integrate metarules into the object grammar but, on the other hand, does not assign them a role in generating an object grammar either, is to view them as redundancy statements describing the relationships that hold among rules in the full grammar. This interpretation eliminates the problem of generating infinite rule sets that gave rise to the Uszkoreit and Peters results. However, it is difficult to see bow the solution supports a computationally useful notion of metarules, since it requires that all rules of the grammar be stated explicitly. Confining the role of metarules to that of stating redundancies prevents their productive application, so that the metarules serve no clear computational purpose for grammar implementation. ${ }^{3}$

We thus conclude that, in contrast to context-sensitive grammars, in which an alternative interpretation of the phrase structure rules makes a difference in weak-generative capacity, MPS grammars do not seem to benefit from the reinterpretations we have investigated.

\section{Formal Constraints}

‥ Je it appears unlikely that a reinterpretation of MPS grammars can be found that solves their complexity problem, formal constraints on the MPS formalism itself have to be explored if we want to salvage the basic concept of metarules. In the following examination of currently proposed constraints, the two criteria for evaluation are their effects on computational tractability and on the explanatory adequacy of the formalism.

As an example of constraints that satisfy the criterion of computational tractability but not that of explanatory adequacy, we examine the issue of essential variables. These are variables in the metarule pattern that can match an arbitrary string of items in a phrase structure rule. Uszkoreit and Peters have shown that, contrary to an initial conjecture by Joshi (see [Gazdar, 1982, fn. 28|), allowing even one such variable per metarule extends the power of the formalism to recursive enumerability. Gazdar bas recommended [1982, p.160] that the power of metarules be controlled by eliminating essential variables, exchanging them for abbreviatory variables that can stand only for strings in a finile and extrinsically determined range. This constraint yieids a computationally tractable system with only context-free power.

Exchanging essential for abbreviatory variables is not, however, as attractive a prospect as it appears at first blush. Uszkoreit and Peters [1982] show that by restricting MPS grammars to using abbreviatory variables only, some significant generalizations are lost. Consider the following metarule that is proposed and motivated in [Gazdar 1982] for endowing VSO languages with the eategory $V P$. The metarule generates tat $\checkmark S O$ sentence rules from $V P$ rules.

(2) $V P \rightarrow V U \Rightarrow S \rightarrow V N P U$

Since $U$ is an abbreviatory variable, its range needs to be stated explicitly. Let us imagine that the VSO language in question has the follow iv $;$ small set of $P$ rules:

(3) $V i$

$$
\begin{aligned}
& V P \rightarrow V N P \\
& V P \rightarrow V \bar{S} \\
& V P \rightarrow V \overline{V P} \\
& V P \rightarrow V N P \overline{V P}
\end{aligned}
$$

Therefore, the range of $U$ has to be $\{\epsilon, N P, \bar{S}, \overline{V P}, N P \nabla P\}$.

\footnotetext{
3 As statements about the object grammar, however, metarules might play $a$ role in language acquisition or in diachronic processes.
} 
If these VP rules are the only rules that satisfy the letthand side of (2), then (2) generates exactly the same rules as it would if we declared $U$ to be an essential rariable-i.e., let its range be $\left(V_{T} \cup V_{N}\right)^{*}$. But now imagine that the language adopts a new subcalegorization frame for verbs, ${ }^{4}$ e.g., a verb that takes an $N P$ and an $\bar{S}$ as complements. $V P$ rule (4) is added:

(4) $V P \rightarrow V N P \bar{S}$

Metarule (2) predicts that $V P_{5}$ beaded by this verb do not have a corresponding fiat VSO sentence rule. We will have to change the metarule by extending the range of $U$ in order to retain the generalization originally intended by the metarule. Obviously, our metarule did not encode the right generalization (a simple intensioa-extension problem).

This shortcoming can also surface in cases where the input to a metarule is the output of another metarule. It might be that metarule (2) not only applies to basic verb rules but also includes the output of, say, a passive rule. The range of the variable $U$ would have to be extended to cover these cases too, and, moreover, might have to be altered if its feeding metarules change.

Thus, if the restriction to abbreviatory variables is to have no effect on the weak-generative capacity of a grammar, the range assigned to each rariable must include the range that would bave actually instantiated the variable on an expansion of the MPS grammar in which the rariable was treated as essential. The assignment of a range to the rariable can only be done pout factum. This would be a satisfactory result, were it not for the fact that finding the necessary range of a variable in this way is an undecidable problem in general. Thus, to exchange essential for abbreviatory variables is to risk affecting the generative capacity of the grammar- - ith quite unintuitive and unpredictable results. In short, the choice is among three options: to affect the language of the grammar in ways that are linguistically unmotivated and arbitrary, to solve an undecidable problem, or to discard the notion of exchanging essential for abbreviatory variables-in effect, a Hobson's choice.

An example of a constraint that satisfies the second criterion, that of explanatory adequacy, but not the first, computational tractability, is the lexical-head constraint of GPSG [Gazdar and Pullum, 1982]. This constraint allows metarules to operate only on rules whose stipulated head is a lexical (preterminal) category. Since the Uszkoreit and Peters results are achieved even under this restriction to the formalism, the constraint does not provide a solution to the problem of expressive power. Of course, this is no criticism of the proposal, since it was never intended as a formal restriction on the class of languages, but rather as a restriction on linguistically motivated grammars. Unfortunately, the motivation behind even this use of the lexicalhead constraint may be lacking. One of the few analyses that relies on the lexical-head constraint is a recent GPSG analysis of coordination and extraction in English [Gazdar, 1981]. In this case-indeed, in general-one could achieve the desired effect

Note that it does not matter whether the grammar writer discovers an additional subcategorization, or the language develops one diachronically; the same problem obtains. simply by specifying that the coefficient of the bar feature be lexical. It remains to be seen whether the constraint must be imposed for enough metarules so as to justify its incorporation as a general principle.

Even with such motivation one might raise a question about the advisability of the lexieal-head constraint on a metatheoretical level. The linguistic intuition behind the constraint is that the role of metarules is to "express generalizations about possibilities of subcategorization" exclusively [Gazdar, Klein, Pullum, and Sag, 1982, p.39], e.g., to express the passive-active relation. This result is said to follow from principles of $\bar{X}$ syntax [Jackendoff, 1977], in which just those categories that are subcategorized for are siblings of a lexical head. However, in a language with freer word order than English, categories other than those subcategorized for will be siblings of lexical heads; they would, thus, be affected by metarules even under the lexical-head constraint. This result will certainly follow from the liberation ruie approach to free word order [Pullum, 1982]. The original linguistic generalization intended by the lexical-head constraint, therefore, will not hold cross-linguistically.

Finally, there is the current proposal of the GPSG community for constraining the formal powers of metarules by allowing each metarule to apply only once in a derivation of a rule. Originally dubbed the once-through hypothesis, this constraint is now incorporated into GPSG under the name finite closure [Gazdar and Pullum, 1982]. Although linguistic evidence for the constraint has never been provided, the formal motivation is quite strong because, under this constraint, the metarule formalism would have only context-free power.

Several linguistic constructions present problems with respect to the adequacy of the finite-closure hypothesis. For instance, the liberation rule teebnique for handling free-word-order languages [Pullum, 1982] would require a noun-phrase liberation rule to be applied twice in a derivation of a rule with sibling noun phrases that permute their subconstituents freely among one another. As a hypothetical example of this phenomenon, let us suppose that English allowed relative clauses to be extraposed in general from noun phrases, instead of allowing just one extraposition. For instance, in this quasi-English, the sentence

(5) Two children are chasing the dog who are small that is bere.

would be a grammatical paraphrase of

(6) Two children wo are small are chasing the dog that is bere.

Let us suppose further that the analysis of this phenomenon involved liberation of the NP $\bar{S}$ substructure of the noun phrases for incorporation into the main sentence. Then the noun-phrase liberation rule would apply once to liberate the subject noun phrase, once again to liberate the object noun phrase. That these are not idle concerns is demonstrated by the following sentence in the free-word-order Australian aboriginal language Warlpiri. ${ }^{5}$

\footnotetext{
${ }^{5}$ This example is taken from [ran Riemsdijk, 1881].
} 
(7) Kurdu-jarra-rlu ka-pala maliki wita-jarra-rlu child-DUAL-ERG AUX:DUAL dog-ABS small-DUAL-ERG yalumpu wajilipi-nyi

that-ABS chase-VONPAST

Two small children are chasing that dog.

The Warlpiri example is analogous to the quasi-English example in that both sentences have two discontinuous $N P$ s in the same distribution. Furthermore, the liberation rale approach has been proposed as a method of modeling the free word order of Waripiri. Thus, it appears that finite closure is not consistent with the liberation rule approach to free word order.

Adverb distribution presents another problem for the hypothesis. In German, for example, and to a lesser extent in English, an unbounded number of adrerbs ean be quite freely interspersed with the complements of a verb. The following German sentence is an extreme example of this phenomenon [Uszkoreit, 1982]. The sequence of its major constituents is giren under (9).

(8) Gestern hatte in der Mittagspause yesterday had during lunch break

der Brigadier in der Werkzeugkammer the foreman (NOM) in the tool shop

dem Lebrling aus Boshattigkeit langram

zebn schmierige Gusseisenscheiben unbemerkt ten greasy cast iron disks (ACC) unnoticed

in die Hosentasche gesteckt

in the pocket put

Yesterday, during lunch break in the tool shop, the foreman, maliciously and unnoticed, put ten greasy cast iron disks slowly into the apprentice's pocket.

(9) ADVP VIN ADVP NPSUBS ADVP NPIOBS ADVP $A D V P N P_{D O B J} A D V P P P V_{I N F}$

A metarule might therefore be proposed that inserts a single adverb in a rerb-phrase rule. Repeated application of this rule (in contradiction to the finite-closure hypothesis) would achieve the desired effeet. To maintain the finite-ciosure hypothesis, we could merely extend the notion of context-free rule to allow reguiar expressions on the right-hand side of a rule. The verb phrase rule would then be accurately, albeit clumsily, expressed as, say, $V P \rightarrow V N P A D V P^{*}$ or $V P \rightarrow$ $V N P A D V P^{*} P P A D V P^{*}$ for ditrangitives.

Similar constructions in free-word-order languages do not permit such naive solutions. As an example, let us consider the Japanese causative. In this construction, the verb suffix "sase" signals the causativization of the verb, allowing an extra $N P$ argument. The process is putatively unbounded (ignoring performance limitations). Furthermore, Japanese allows the NPs to order freely relative to one another (subjeet to considerations of ambiguity and focus), so that a flat structure with some kind of extrinsic ordering is presumably preferable.

One means of achieving a flat structure with extrinsic ordering is by using the ID/LP formalism, a subformalism of
GPSG that allows immediate dominance (ID) informaticn to be specified separately from linear precedence (LP) zotions. (CP. context-free phrase structure grammar, wbich forces a strict oneto-one correlation between the two types of informstion.) ID information is specified by context-free style rules ith unordered right-band sides, notated, e.g., $A \rightarrow B, C, D$. LP information is specified as a partial order over the nonterminals in the $4 \cdot \mathrm{mm}$ mar, notated, e.g., $B<C$ (read $B$ precedes $C$ ). These two rules can be viewed as schematizing a set of three context-free rules, namely, $A \rightarrow B C D, A \rightarrow B D C$, and $A \rightarrow D B C$.

Without a causativization metarule that can operate more than once, we might attempt to use the regular expression notation that solved the adverb problem. For example, we might postulate the ID rule $V P \rightarrow N P^{*}, V$, sase with the LP relation $N P<V<$ sase, but no matching of $N P s$ with sases is achieved. We might attempt to write a liberation rule that pulls NP-sase pairs from a nested structure into a flat one, but this would violate the finite-closure hypothesis (as well as Pullum's requirement precluding liberation through a recursive category). We could attempt to use even more of the power of regular-expression rules with $\mathrm{ID} / \mathrm{LP}$, i.e., $V P \rightarrow\{N P, \text { sase }\}^{*}, V$ under the same LP relation. The formalism presupposed by this analysis, bowever, bas greater than context-free power, ${ }^{B}$ so that this solution may not be desirable. Nevertheless, it should not be ruled out belore the parsing properties of such a formalism are understood.? Gunji's analysis of Japanese, which attempts to solve such problems with the muitiple application of a slash introduction metarule [Gunji, 1980], again raises the problem of violating the finite-closure bypotbesis (as well as being incompatible with the current version of GPSG which disallows multiple slashes). Finally, we could always move causativization into the lexicon as a lexical rule. Such a move, though it does circumvent the dificulty in the syntax, merely serves to move it elsewhere without resolving the basic problem.

Yet another alternative involves treating the right-hand sides of phrase structure rules as sets, rather than multisets as is implicit in the ID/LP format. Since the nonterminal rocabulary is finite, right-hand sides of ID rules must be subsets of a finite set and therefore finite sets themselves. This bypothesis is quite similar in effect to the finite-closure hypothesis, albeit even more limited, and thus inherits the same problems as were discussed above.

\section{The Ultimate Solution}

An obvious way to constrain MPS grammars is to eliminate metarules entirely and replace them with other mechanisms. In fact, within the GPSG paradigm, sereral of the functions of metarules have been replaced by other metagrammatical devices. Other functions have not, as of the writing of this paper, though

\footnotetext{
${ }^{6}$ For instance, the grammar $S \rightarrow\{a, b, c\}^{*}$ with $a<b<c$ generates $a^{n} b^{n} c^{n}$.

${ }^{7}$ Shieber [fortheoming) provides an algorithm for parsing DD/LP grammars directly that includes a method for utilizing the Kleene star derice. It could be extended to even more of the regular expression notation, though the effect of such extension on the time complexity of the algorithm is an open question.
} 
it is instructive to cocsicer the esses covered by this class. In the discussion to follow we bave isolated taree of the primary functions of metarules. This is not intended as an exhaustive taxonomy, and certain metarules may manifest more than one of these functions.

First, we consider generalizations over linear order. If metarules are metagrammatical statements about rules encoding linear order, they may relate rules that differ only in the linear order of categories. With the introduction of ID/LP format, however, the hypothesis is that this latter metagrammatical device will suffice to account for the linear order among the categories within rules. For instance, the problematic adverb and causative metarules could be replaced by extended context-free rules with ID/LP, as was suggested in Section 3 above. Shieber [forthcoming) has shown that a pure ID/LP formalism (witbout metarules, Kleene star, or the like) is no less computationally tractable than context-free grammars themselves. Although we do not yet know what the consequences of incorporating the extended context-free rules would be for computational complexity, ID/LP format can be used to replace eertain word-ordervariation metarules.

A second function of metarules was to relate sets of rules that differed only in the values of certain specifed features. It has been suggested [Gazdar and Pullum 1082] that such features are distributed secording to certain general principles. For instance, the slash-propagation metarule has been replaced by the distribution of slash features in accord with such a principle.

A third function of metarules under the original interpretation bas not been relegated to other metagrammatical devices. We bare no single device to suggest, though we are exploring alternative ways to account for the phenomena. Formally, this third class can be characterized as comprising those metarules that relate sets of rules in which the number of categories on the right- and left-hand sides of rules differ. It is this sort of metarule that is essential for the extension of GPSGs beyond context-free power in the Uszkoreit and Peters proois [1982). Simply requiring that such metarules be disallowed would not resolve the linguistic issues, however, since this constraint would inherit the problems connected with the regular expression and set notations discussed in Section 3 above. This third class further breaks down into two cases: those that have different parent categories on the right and left-hand sides of the metarule and those that have the same category on both sides. The first case includes those liberation rules that figure in analyses of free-word-order phenomena, plus such other rules as the subject-auxiliary-inversion metarule in Engtish. Uszkoreit [forthcoming] is exploring a method for isolating liberation rules in a separate metagrammatical formalism. It also appears that the subject-auxiliary inversion may be analyzed by alrcady existing principles governing the distribution of features. The second case (those in wich the categories on the right and left-hand sides are the same) includes such analyses as the passive in English. This instance, at least, might be replaced by a lexical-redundancy rule. Thus, no uniform solution has yet been found for this third function of metarules.

We conciude that it may be possible to replace MPS-style metagrammatical formalisms entirely without losing generaliza-

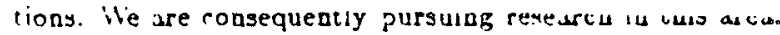

\section{Conclusion}

The formal power of metarule formalisms is clearly an important consideration for computational linguists. Uszkoreit and Peters [1982] have show a that the potential exists for defining metarule formalisms that are computationally "unsafe." However, thesé results do not sound a death knell for metarules. On the contrary, the safety of metarule formalisms is still an open question. We have merely show that the constraints on metarules necessary to make them formally tractable will have to be based on empirical linguiatic evidence as well as solid formal research. The solutions to constraining metarules analyzed bere seem to be either formally or linguistically inadequate. Further research is aeeded in the actuai uses of metarules and in constructions that are problematic for metarules so as to deveiop either linguistically motivated and computationally interesting constraints on the formalisms, of alternative formalisms that are linguistically adequate but not beir to the problems of metarules.

\section{References}

Gawron. J. M., et al., 1082: “Processing English with a Generalized Phrase Structure Grammar," in Proceedings of the eoth Annual Heeting of the Association for Computational Linguistics, University of Toronto, Toronto, Canada (16-18 June).

Gazdar, G., 1282: "Phrase Structure Grammar," in P. Jacobson and G. Pullum, eds., The Nature of Syntactic Representation (Reidel, Dordrecht, Holland).

Gazdar, G.. E. Klein, G.K. Pullum, and I.A. Sag, 1982: "Coordinate Structure and Unbounded Dependencies," in M. Barlow, D.P. Fliekinger, and t.A. Sag, eds., Devedopments in Generadized Phrase Strueture Grammar, Stanford Working Papers in Grammatieal Theory, Volume 2 (Indiana University Linguisties Club, Bloomington, Indiana, November).

Gazdar, G. and G.K. Pullum, 1981: "Subeategorization, Constituent Order and the Notion 'Head'," in M. Moortgat, H.v.d. Hulst and T. Hockstra. eds., The Scope of Lerical Rules, pp. 107-123 (Foris, Dordreeht, Holland).

Gazdar, G. and G.K. Pullum, 1982: "Generalized Phrase Strueture Grammar: A Theoretical Synopsis," (Indiana University Linguisties Club, Bloomington, Indiana, August).

Gazdar, G.. G.K. Pullum, and I.A. Sag, 1982: “Auxiliaries and related phenomena." Langrage, Volume 58. Number 3, pp.591-638.

Gunji, T., 1980: "A Phrase Structure Analysis of the Japanese Language," M. A. dissertation, Ohio State University, Columbus, Ohio.

Jackendoff, R., 1077: “ $\bar{X}$ Synt2x," Linguistic Inquiry Monograph 2, (MIT Press, Cambridge, Massachusetts).

Kay, M., 1982: "When Meta-Rules are Not Meta-Rules," in M. E.arlow. D.P. Flickinger, and I.A. Sag, eds., Developments in Generalized Phrose Structure Grommor, Stanford Working 
Papers in Grammatical Theory, Volume 2 (Indiana University Linguistics Club, Bloomington, Indiana, November).

Peters, S. and R.W. Ritchie, 1973: "Context-Sensitive Immediate Constituent Analysis: Context-Free Languages Revisited," in Mathematical Syutems Theory, Vol. 6, No. 4, pp. 324-333 (Springer-Verlag, New York).

Peters, S. and R.W. Ritchie, fortheoming. "Phrase-Linking Grammars."

Pullum, G.K., 1982: "Free Word Order and Phrase Structure Rules," J. Pustejoviky and P. Sells, eds., Proceedings of the Twelfts Annual Meeting of the North Eaotern Linguistic Society, (Graduate Linguistics Student Association, University of Massachusetts, Amherst, Massachusetts).

Shieber, S., forthcoming: "Direet Parsing of ID/LP Grammars."

Stucky, S., fortheoming: "Metarules as Meta-NodeAdmissibility Conditions."

Thompson, H., 1982: "Handling Metarules in a Parser for GPSG," in M. Barlow, D.P. Fliekinger, and I.A. Sag, eds., Developmenls in Generadized Phrase Structure Grammar, Stanford Working Papers in Grammatical Theory, Volume 2 (Indiana University Linguistics Club, Bloomington, Indiana, November).

Uszkoreit, H., forthcoming: "Constituent Liberation."

Uszkoreit, H. and S.J. Peters, 1982: “Essential Variables in Metarules," presented at the 1082 Annual Meeting of the Linguistic Society of America, San Diego, California (December).

van Riemsdijk, H., 1981: "On 'Adjacency' in Phonology and Syntax," in V.A. Burke and J. Pustejovsky, eds., Proceedings of the Eleventh Annual Meeting of the North Eosiern Linguistic Society, University of Massachusetts, Amherst, Massachusetts, pp. 390 413 (April). 